\title{
Innate Color Preference of Zebrafish and Its Use in Behavioral Analyses
}

\author{
Jong-Su Park ${ }^{1,4}$, Jae-Ho Ryu ${ }^{1,4}$, Tae-Ik Choi ${ }^{1}$, Young-Ki Bae ${ }^{2}$, Suman Lee ${ }^{3}$, Hae Jin Kang ${ }^{1, *}$, and Cheol-Hee \\ $\mathrm{Kim}^{1, *}$
}

\begin{abstract}
Although innate color preference of motile organisms may provide clues to behavioral biases, it has remained a longstanding question. In this study, we investigated innate color preference of zebrafish larvae. A cross maze with different color sleeves around each arm was used for the color preference test (R; red, G; green, B; blue, Y; yellow). The findings showed that $5 \mathrm{dpf}$ zebrafish larvae preferred blue over other colors $(B>R>G>Y)$. To study innate color recognition further, tyrosinase mutants were generated using CRISPR/Cas9 system. As a model for oculocutaneous albinism (OCA) and color vision impairment, tyrosinase mutants demonstrated diminished color sensation, indicated mainly by hypopigmentation of the retinal pigment epithelium (RPE). Due to its relative simplicity and ease, color preference screening using zebrafish larvae is suitable for high-throughput screening applications. This system may potentially be applied to the analysis of drug effects on larval behavior or the detection of sensory deficits in neurological disorder models, such as autism-related disorders, using mutant larvae generated by the CRISPR/Cas9 technique.
\end{abstract}

\section{INTRODUCTION}

Color vision is one of the most important modalities in the recognition of biologically important stimulation, and thus it plays a critical role in visual perception. Depending on the number of cone types, animals including humans can perceive colors based on the distribution of the wavelengths of light. Humans and some primates have trichromatic vision, but most non-primate mammals are dichromatic; many lower vertebrates,

${ }^{1}$ Department of Biology, Chungnam National University, Daejeon 34134, Korea, ${ }^{2}$ Comparative Biomedicine Research Branch, National Cancer Center, Goyang 10408, Korea, ${ }^{3}$ Division of Structural and Functional Genomics, Center for Genome Science, National Research Institute of Health, Osong 28159 , Korea, ${ }^{4}$ These authors contributed equally to the work.

*Correspondence: haejin31@gmail.com (HJK); zebrakim@ cnu.ac.kr (CHK)

Received 12 July, 2016; revised 22 September, 2016; accepted 12 October, 2016; published online 31 October, 2016

Keywords: autism, behavioral analysis, color blindness, color preference, zebrafish such as birds and fish, are tetrachromatic. Phylogenetic analysis of opsin gene sequences suggests that cones were the ancestral pigments with rods evolving afterward. It is yet to be thoroughly investigated, but the nocturnal bottleneck hypothesis explains this counterintuitive fact (Bowmaker, 2008; Gerkema et al., 2013; Jacobs, 2009; Robertson et al., 2004) (Fig. 1). Many groups point to the possibility that, although most mammals lack one or more opsin when compared to fish and primates, the rest of the machinery and strategies used in color processing were not lost through evolution (Joselevitch and Kamermans, 2009).

Innate color preference or aversion aids in the recognition of one's environment and can provide information on how to appropriately respond to environmental stimuli. However, limited research has been conducted in this area. In humans, genderstereotyped color preference was reported in a study involving young female subjects who demonstrated increasing attraction to pink over blue, whereas male counterparts displayed growing avoidance of pink (LoBue and DeLoache, 2011). Colors within the visible spectrum are determined by the wavelength of light, thus color recognition relies on the cones of the retina. Zebrafish (Danio rerio) possess eyes and retina that are similar to those of other vertebrates including humans (Glass and Dahm, 2004). The cell layout of zebrafish retina closely resembles that of the human retina. Importantly, zebrafish have a rich color vision, which is a distinct advantage over the rodent models (Goldsmith and Harris, 2003). Comparable to humans, zebrafish possess the UV-cone (362 nm), S-cone (417 nm), M-cone (480 nm), and Lcone $(556 \mathrm{~nm})$ (Nawrocki et al., 1985). Zebrafish eyes detect light by $3.5 \mathrm{dpf}$, and zebrafish larva displays mobility starting from 5 dpf. Based on this information, an innate color preference test can be designed to assay as soon as zebrafish larvae begin to swim. This arrangement ensures protection from all color stimuli until experiments begin, which negates postnatal color adaptation. One previous color preference study showed that adult zebrafish have a strong aversion towards blue compared to red, green, and yellow colors. Avdesh et al. (2012) suggested that zebrafish ( 25 weeks) have natural color preferences without any rewarded stimuli. However, innate color preference studies of laboratoryavailable fishes, such as zebrafish, guppies, and medaka, have not yet received ample attention in the scientific literature.

Oculocutaneous albinism (OCA) is a group of inherited disorders characterized by defective melanin biosynthesis which leads to a reduction or a complete lack of melanin pigment in the hair, skin, and eyes. Clinical manifestations of OCA include reduced pigmentation of the RPE and impaired color vision.

eISSN: 0219-1032

(c) The Korean Society for Molecular and Cellular Biology. All rights reserved.

(c) This is an open-access article distributed under the terms of the Creative Commons Attribution-NonCommercial-ShareAlike 3.0 Unported License. To view a copy of this license, visit http://creativecommons.org/licenses/by-nc-sa/3.0/. 


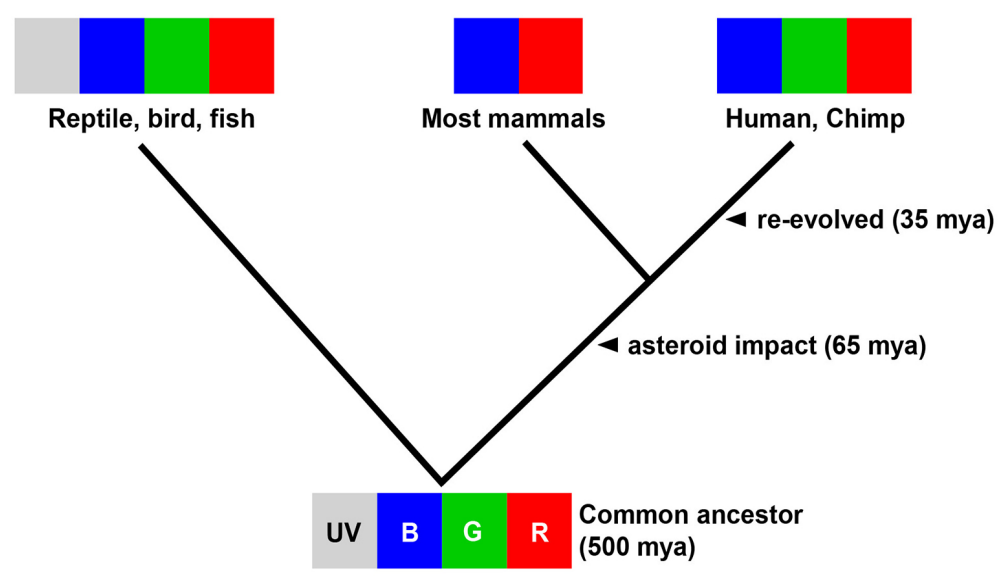

Fig. 1. Photoreceptor evolution in vertebrates. The common ancestor of tetrapods and amniotes are tetrachromats. Asteroid impact at 65 million years ago (mya) led to the Cretaceous-Paleogene extinction event when most mammals became dichromates to adjust to underground habitats. 'Reevolution' occurred around 35 mya when primates became trichromats (Bowmaker, 2008; Jacobs, 2009).
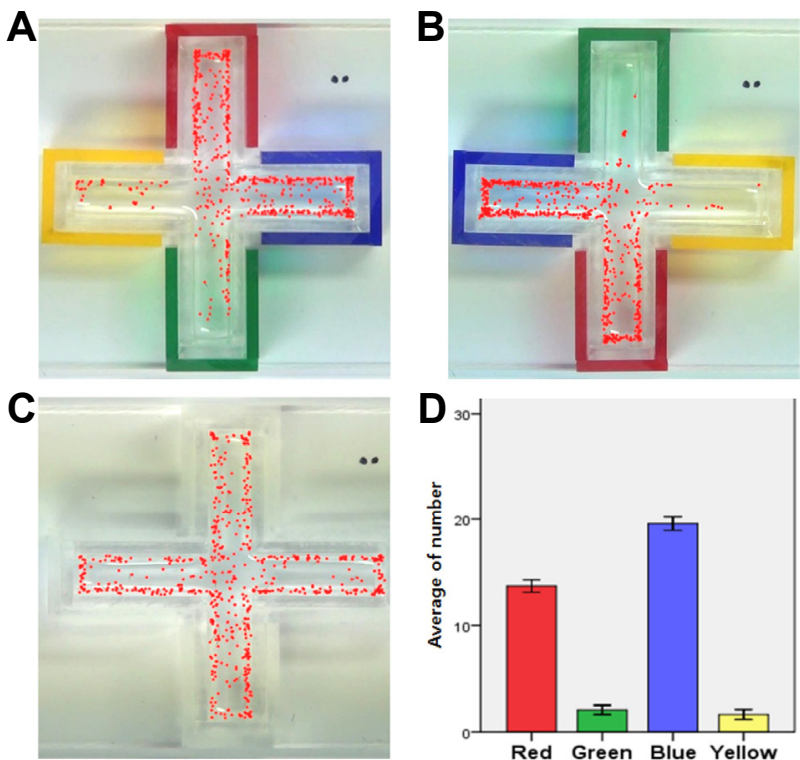

Fig. 2. Cross color maze and preference tests. (A-C) The location of larvae $(n=40)$ is marked as red dots every 2 min for 30 min in the cross color maze with RGBY color sleeves $(A, B)$ and no color sleeves (C). (D) The average number of larvae in each arm (Right, Left, Upper, Below) for 30 min was graphed $(n=40)$.

Tyrosinase is the rate-limiting enzyme involved in melanin biosynthesis. Abnormal tyrosinase caused by errors occurred during post-translational modification or trafficking into melanosomes can decrease pigmentation in skin and RPE (Grønskov et al., 2007). Previous studies demonstrated that the CRISPR/Cas9 system can be used to efficiently induce a sitespecific modification in zebrafish (Singh and Nüsslein-Volhard, 2015). CRISPR/Cas9 system makes highly specific cuts in DNA molecules and carries out insertions or deletions at the sequence sites.

Zebrafish display a broad spectrum of behaviors such as learning, cognition, aggression, anxiety and social interaction (Fadool and Dowling, 2008; Miller et al., 2015; Orefice et al., 2016; Richendrfer et al., 2012). With the innate color preference test, we have a simple way to measure quantitative effects of small molecules and observe behavioral changes due to blindness or neurodevelopmental disorders at a very early larval stage. To understand the molecular mechanisms underlying animal behaviors, we established an in vivo knockout zebrafish model utilizing the CRISPR/Cas9 system (Lee, 2016; May et al., 2015; Sung et al., 2014).

\section{MATERIALS AND METHODS}

Ethics statement

All experiments using zebrafish were performed in accordance with the Institutional Animal Care and Use Committees of Chungnam National University (CNU-00393).

\section{Animals and housing}

Wild-type zebrafish were purchased from local pet stores (Korea) and used as breeders. 50 100 fertilized eggs were raised in $50 \mathrm{ml}$ egg water (sea salt $60 \mathrm{ug} / \mathrm{ml}$ ) in a transparent $90 \mathrm{~mm}$ culture dish (10091, SPL, Korea) at $28.5^{\circ} \mathrm{C}$ with a $14 \mathrm{~h}$ light on/ $10 \mathrm{hr}$ light off cycle under approximately 3000 Lux fluorescent light. After hatching, larvae swam freely without any disturbance until $7 \mathrm{dpf}$, then reared as previously described (May et al., 2015). A combination of granule (Hatchfry Encapsulon grade 0 , Argent Lab, USA) and brine shrimp was fed from $8 \mathrm{dpf}$, and then switched to brine shrimp from $16 \mathrm{dpf}$.

\section{Preparation of sgRNA and Cas9} tyrosinase (ENSDARG00000039077) target sites of Cas9 were identified using the ZiFiT Targeter (http://zifit. partners.org/ ZiFiT/) and selected oligonucleotides were cloned into pDR274 (Addgene) linearized with Bsa I (BioLabs). The templates for in vitro transcription of sgRNAs were produced by PCR using primers 5' TAGGACTGGAGGACTTCTGGGG 3' (oligo \#1) and 5' AAACCCCCAGAAGTCCTCCAGT 3' (oligo \#2). In vitro transcription was carried out using 150-200 ng template and the MaxiScript T7 Kit (Ambion). RNA was precipitated with isopropanol. Cas9 expression vector (from Addgene) was linearized with Dra I (Takara) and purified with an agarose gel DNA extraction kit (ELPIS). Cas9 mRNA was transcribed with the mMESSAGE mMACHINE ${ }^{\circledR}$ T7 Kit (Ambion) poly (A) tailed with E. coli Poly (A) Polymerase (NEB) and then purified by lithium chloride precipitation following the manufacturer's protocol. 
A

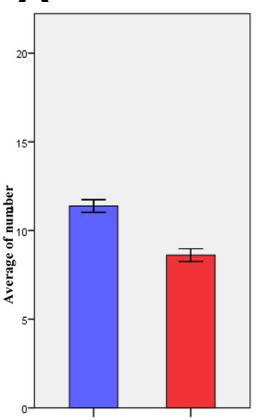

B $\quad \mathbf{R}$

F

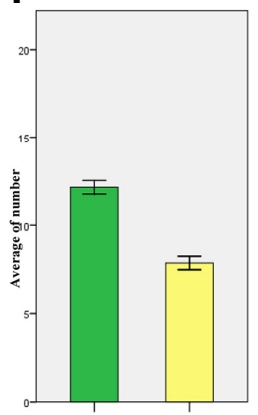

G
B

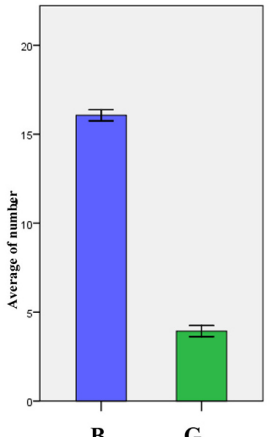

G

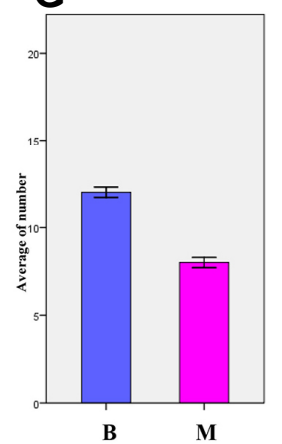

C

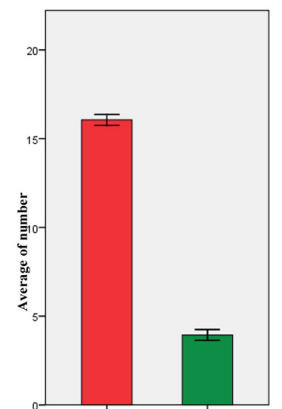

G
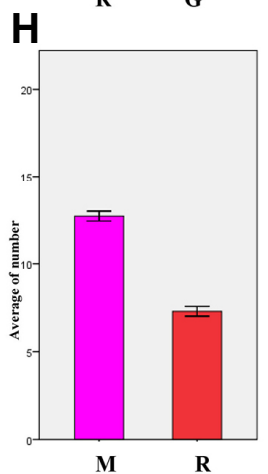

D

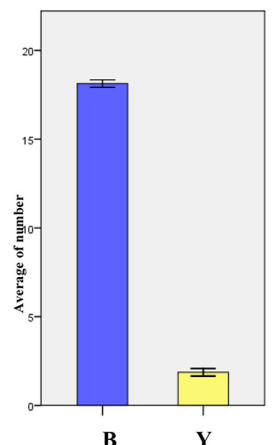

I

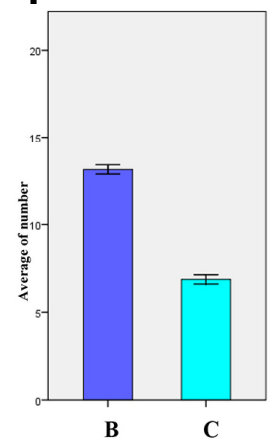

E

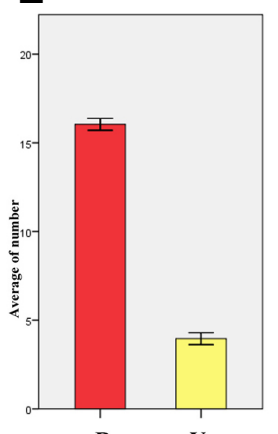

J

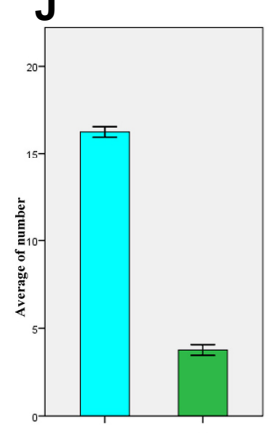

Fig. 3. Color preference tests on the bidirectional maze with 6 colors (RGBYMC). The average numbers of larvae $(n=20)$ at each color arm was counted every $2 \mathrm{~min}$ for a total of $30 \mathrm{~min}$. R, red; G, green; B, blue; Y, yellow; M, Magenta; C, Cyan. (A) B to R color preference. (B) B to $G$ color preference. (C) $R$ to $G$ color preference. (D) B to $Y$ color preference. (E) $R$ to $Y$ color preference. (F) $G$ to $Y$ color preference. (G) B to $M$ color preference. (H) M to R color preference. (I) B to $C$ color preference. (J) $C$ to $G$ color preference.

\section{Microinjection and genotyping}

One-cell stage zebrafish embryos were injected with $350 \mathrm{ng} / \mu \mathrm{l}$ Cas9 mRNA and $100 \mathrm{ng} / \mathrm{\mu l}$ sgRNA. tyr sgRNA and Cas9 mRNA injected embryos shows $70-80 \%$ mutation at tyr loci. PCR primers for the genotyping of the zebrafish tyr mutant are, forward primer, 5' GCGTCTCACTCTCCTCGACTCTTC 3' and reverse primer, 5' GTAGTTTCCGGCGCACTGGCAG 3'. PCR products $(20 \mu \mathrm{l})$ were re-annealed in a thermal cycler under the following conditions: $95^{\circ} \mathrm{C}$ for $2 \mathrm{~min}, 95^{\circ} \mathrm{C}$ to $85^{\circ} \mathrm{C}$ at $2^{\circ} \mathrm{C} / \mathrm{s}, 85^{\circ} \mathrm{C}$ to $25^{\circ} \mathrm{C}$ at $0.1^{\circ} \mathrm{C} / \mathrm{s}$, then kept at $4^{\circ} \mathrm{C}$. The $16 \mu \mathrm{l}$ of the re-annealed mixture was incubated with $0.2 \mu$ of T7 endonuclease I, $2 \mu \mathrm{l}$ of NEB buffer 2 and $1.8 \mu \mathrm{l}$ of NFW (Nuclease-free water) at $37^{\circ} \mathrm{C}$ for $40 \mathrm{~min}$. To screen F2 homozygous mutant, PCR was performed using forward primer, 5' GCGTCTCACTCTCCTCGACTCTTC 3' and reverse primer, 5' TCGCCGGGCCAGACTGGACAGCA 3'.

\section{Color preference test with cross and bidirectional maze}

Color preference test was performed either in a cross (Figs. 2 and $6 \mathrm{~B}$ ) or bidirectional color maze (Figs. 3 and 6A). The maze was built using $3 \mathrm{~mm}$ clear acrylic sheets. The cross maze had four arms, and the bidirectional color maze had two arms in a transparent acryl plate. The dimension of each arm was $10(\mathrm{~W})$ $\times 35(\mathrm{~L}) \times 15(\mathrm{H}) \mathrm{mm}$. The maze was left uncovered on top. Different color sleeves could be placed on the outside of each arm to provide different color cues $(\mathrm{N}$; no color sleeve, $\mathrm{R}$; red, G; green, B; blue, Y; yellow, M; magenta, C; cyan). The maze was implemented to have 20 zebrafish larvae (from 4 dpf to 30 dpf) freely swimming in all direction. With color sleeves, zebrafish larvae $(n=40)$ swam freely during the 30 min experiment. The location of each larva was indicated with a red dot every 2 min for a total duration of 30 min of video recording (Fig 2 ), and the number of larvae in each arm was manually counted. To eliminate any unexpected bias, color sleeves were moved to opposite arms after each experiment.

\section{Measurement of wavelength vs. lighting intensity}

The transmitted wavelength spectrum of all color sleeves and stand light were measured using a spectrometer (SV2100-VIS, $\mathrm{K}-\mathrm{Mac}$, Korea). In the penetration wavelength spectrum measurement, a commercial stand light with a typical cool white fluorescent lamp (FPL27EX-D, Jianxi South Lightings, China) was used as a light source. Light intensity was measured with a lux meter (TES-1335, TES, Taiwan). The spectrum of the lamp had main peaks including red $(607 \mathrm{~nm})$, green $(546 \mathrm{~nm})$ and blue $(435 \mathrm{~nm})$ wavelengths. Yellow is comprised of red and green. Magenta is comprised of blue and red. Cyan is comprised of blue and green.

Image processing, video tracking and statistical analysis All maze experiments were recorded using a high-resolution digital camera (HDR-CX130, Sony, Japan). The video file was first stored on the camera's memory card and subsequently transferred to the computer's hard drive. The full-HD video file that was produced in the MTS format from the digital camera was approximately 2 gigabyte/file. The number of zebrafish 
A

$\mathbf{A}_{\text {GTCTGCACCTCCCCAGAAGTCCTCCAGTCCAAACG }}$

GTCTGCACCACAGACTCCACAGACTGGAGGACAGAAGTCCTCCAGTCCAAACG

GTCTGCACCTCCTCCAGAAGTCCTCCAGTCCAAACG
Wild Type

(-4 del, +22 insertion)

(+1 insertion)

B
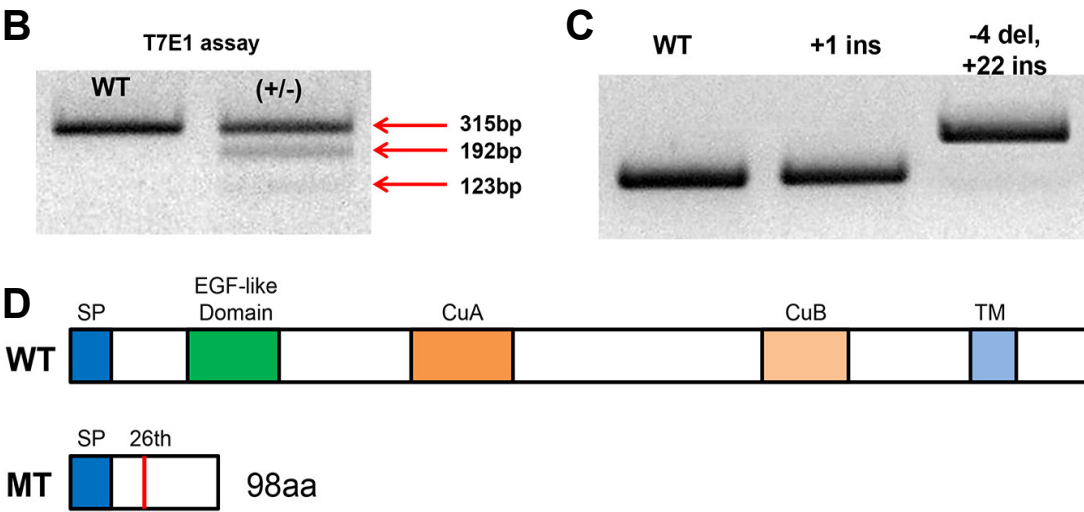

$98 a a$
Fig. 4. Generation of tyrosinase mutant zebrafish by CRISPR/Cas9. (A) Two alleles of $\mathrm{F} 1$ heterozygous mutants $(+18,+1$ insertion) were acquired. (B) F1 heterozygous mutants were confirmed using T7 endonuclease I. (C) Homozygous mutant F2 generation shows shifted band. (D) WT protein consists of SP, EGF-like Domain, CuA, CuB, TM. KO \#2 mutants fish (+1) showed one base insertion at base 78 in exon 1 (c.78/79 insT, 26 $6^{\text {th }}$ amino acid), which generated premature stop at $98^{\text {th }}$ amino acid. SP - Signal peptide, EGF-like domain - Epidermal growth factor-like domain, CuA, CuB - Copper binding domain $A, B, T M$ - Transmembrane domain. larvae $(n=40)$ in each color zone was counted manually every 2 minutes for $30 \mathrm{~min}$ (Fig. 2). To compare the Wild-type and tyrosinase mutant test, duration times were checked in each color sleeves during the $30 \mathrm{~min}$ experiment (Fig. 6). All these video tracking experiments were performed using Danio vision powered by Etho visionXT ${ }^{\circledR}$ (Noldus, Netherland) to track zebrafish (Singh and Nüsslein-Volhard, 2015).

\section{Histology (Paraffin section \& H\&E staining)}

For paraffin embedding, embryos were dehydrated with ethanol for $5 \mathrm{~min}$ and washed for $10 \mathrm{~min}$ in isopropanol. They were then cleared three times for $10 \mathrm{~min}$ in Xylene. Embryos were transferred to xylene:paraffin $(1: 1)$ solution at $60^{\circ} \mathrm{C}$ for $20 \mathrm{~min}$ and then embedded three times for 20 min with paraffin in a stainless steel mold. Embryos were held in position in the prewarmed plastic embedding cassette. After trimming, sections (8 $\mu \mathrm{m})$ were cut by a microtome and deparaffinized with Xylene. The preparations were mounted with Canada balsam and examined by LEICA DM5000B microscope.

\section{Data presentation}

All data and graphs were expressed as mean \pm standard error of the mean (SEM) and analyzed by an independent t-test using the statistics program (SPSS, IBM, USA). All figures were adjusted for size using Photoshop.

\section{RESULTS}

Color discrimination of zebrafish larva in RGBY color cross maze

A cross maze with four arms was developed as a four color preference test (Fig. 2). To test RGBY color preference, the cross maze was fitted with color sleeves (R, G, B, and $Y$ ) placed one on each arm, and $5 \mathrm{dpf}$ zebrafish larvae $(n=40)$ were introduced and allowed to swim freely. The number of larvae in each arm was counted every 2 min for $30 \mathrm{~min}$ total (Figs. 2A and 2B). In the control experiment without color sleeves, the larvae were uniformly distributed (Fig. 2C); how- ever, experimental data showed that more larvae preferred to stay in $B(19.57 \pm 1.76)$ and $R$ zones $(13.70 \pm 1.60)$ than in $G$ and $Y$. Thus, innate color preference of zebrafish larvae was determined as $B>R>G>Y$. This color test showed that zebrafish larvae exhibited both color discrimination and distinct color preference. To eliminate group effect, a single larva was tested in a bidirectional color maze and the same color preference was again observed (data not shown). Also, pre-exposure to color stimulus $(B, Y)$ did not alter innate color preference of zebrafish larvae (data not shown).

Color preference test on bidirectional maze for two color comparisons

A bidirectional maze was developed to compare color preference between two colors chosen from R, G, B, Y, C or M. This test yielded a similar result to that of the color cross maze larvae preferred $B$ and $R$ over $G$ and $Y$, and the order of $R G B Y$ preference was $B>R>>G>Y$ (Fig. 3).

Between the primary $(\mathrm{R} / \mathrm{G} / \mathrm{B})$ and secondary $(\mathrm{Y})$ colors, larvae showed a greater preference for $B$ and $R$ over $Y$ (Figs. 3D and $3 E)$. Larva also had a tendency to stay more in the $G$ zone $(12.14 \pm 0.194)$ than in the $Y$ zone $(G>Y)(7.86 \pm 0.194)(P<$ $0.001)$. In the case of magenta, $B(12.00 \pm 0.148)$ was preferred over $M(8.00 \pm 0.148)$, and $M(12.71 \pm 0.140)$ was preferred over $R(7.29 \pm 1.536)$, suggesting a color preference of $B$ $>M>R$. In the case of cyan, $B(13.13 \pm 0.133)$ was preferred over $C(6.87 \pm 0.133)$, and $C(16.24 \pm 0.152)$ was preferred over $\mathrm{G}(3.76 \pm 0.152)$, and suggested an order of $\mathrm{B}>\mathrm{C}>\mathrm{G}$. No distinct preference was observed between $R$ and $C$. Overall, the order of RGBYMC preference was $B>M>R, C>M>Y$.

Generation of color blindness models in zebrafish by CRISPR/Cas9

OCA is a heterogeneous autosomal recessive genetic disorder of melanin synthesis. OCA results in reduced or absent pigmentation in the hair, skin, and eyes. In the eye, a pigmented cell layer called RPE shields the neurosensory retina from excess incoming light. Reduced pigmentation of RPE and color 


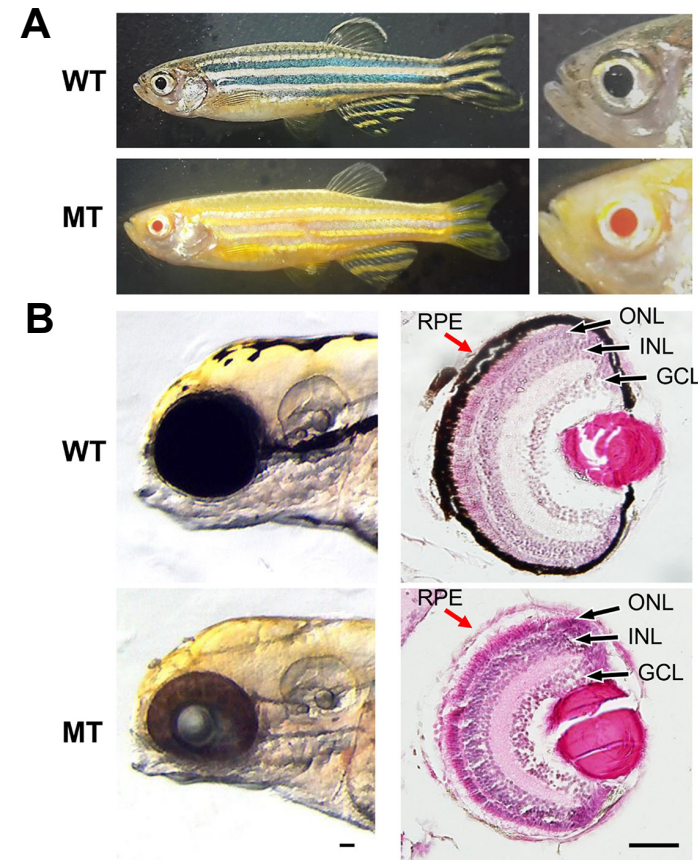

Fig. 5. Comparison of WT, MT adult zebrafish phenotypes and histological analysis of $5 \mathrm{dpf}$ zebrafish larva. (A) The $3 \mathrm{mpf}$ adult zebrafish WT display golden and blue horizontal stripes and mosaics of yellow xanthophores, silvery or blue iridophores, and black melanophores but MT show only golden horizontal stripes and red eyes. (B) Histological analysis of WT and MT larvae at $5 \mathrm{dpf}$ MT lacks black melanin pigment in RPE (red arrow) compared to WT. dpf - days post fertilization, mpf - month post fertilization, RPE retinal pigment epithelium, GCL - ganglion cell layer, INL - inner nuclear layer, ONL - outer nuclear layer. Scale bars, 50 um.

vision impairment are known symptoms of OCA patients. Among many types of OCA, type 1 OCA (OCA1) occurs as a result of tyrosinase (tyr) gene mutation (Liu et al., 2014). Zebrafish tyr mutants that mimic the condition of oculocutaneous albinism in humans were generated using the CRISPR/Cas9 system. Mutations were confirmed by T7El assay, and two homozygous mutant lines were established. Genomic DNA sequencing analysis of the $\mathrm{KO} \# 1$ mutant showed a 22 base pair (bp) insertion and 4 bp deletion, resulting in an $18 \mathrm{bp}$ in-frame mutation; homozygous KO \#1 fish exhibited normal development of melanocytes. Genomic DNA sequencing analysis of $\mathrm{KO} \# 2$ mutant revealed a 1 bp insertion at base 78 in exon 1 (Fig. 4A). This alteration led to the premature termination of Tyr protein during translation (Fig. 4D). The adult KO \#2 mutant zebrafish exhibited albinism phenotypes, having a golden body and red eyes due to the absence of black melanophores (Fig. 5A) (Singh and Nüsslein-Volhard, 2015). Histological analysis of the eyes of 5dpf, WT, and Tyr-mutant zebrafish demonstrated reduced eye size and hypo-pigmented RPE in mutant fish. The structure of the retina was normal in Tyr mutant zebrafish, however, the RPE lacked pigment (Fig. 5B).

Color preference tests in zebrafish color blindness models The color preference test of tyr mutant larvae was performed with a bidirectional maze and color cross maze. Colors $B$ and $Y$ were compared in the bidirectional maze and $B, R, G$ was compared in the color cross maze. In each experiment, a single larva was tracked for 30 min using Etho visionXT ${ }^{\circledR}$ (Noldus, Netherland).

The data showed WT larvae preferred $B$ over $Y$ in the bidirectional maze and spent more than $20 \mathrm{~min}$ in the $\mathrm{B}$ zone during the 30-min experiment. On the other hand, tyr mutant larvae did not show any color preference, staying equal amounts of time in each arm (Fig. 6A). Likewise, WT larvae showed $\mathrm{B}>\mathrm{R}>\mathrm{G}$ preference in the color cross maze while tyr mutants demonstrated no color preference between blue, red and green but tended to stay longer in the green zone (Fig. 6B). Taken together, the data suggests that tyr knockout mutants cannot distinguish color which is also the case for human OCA patients.

\section{DISCUSSION}

In pharmaceutical studies, zebrafish larvae have recently been utilized in high-throughput applications to determine the quantitative effects of small molecules on rest, wake, motor behavior and cognition (Miller et al., 2015; Richendrfer et al., 2012). This study examined innate color preference using zebrafish larvae. Such investigations involving innate color recognition and response should be designed to avoid postnatal adaptation and address the subtle responses to monochromatic light in daytime (Sugimoto et al., 2005). During the course of our research, Peeters et al. (2016) also reported on the color preference of zebrafish. The findings showed that normal lavae preferred blue and avoided yellow. When we tested primary and secondary colors, zebrafish larvae preferred $B$ over $M$, and $M$ over $R$. The same tendency was observed in $B, C$, and $G$ - B was preferred over $C$ and $C$ was preferred over $G$; however $Y$ was avoided in all cases. This phenomenon was consistent throughout the experiment despite maze shape, suggesting that zebrafish larvae are instinctively attracted to blue and avoided yellow which is the complement of blue. Environmental factors such as light intensity or pre-exposure to colors did not affect innate color preference, and the number of tested larvae (1 40 larvae) was not shown to affect the result, negating group effect (Supplementary Fig. 1).

To further test innate color preference of zebrafish larvae, a color blindness model (OCA-like zebrafish) was generated using the CRISPR/Cas9 system. tyrosinase KO \#2 mutant fish had a $1 \mathrm{bp}$ insertion in exon 1 which caused a premature termination of tyrosinase protein. Given that tyrosinase controls the production of melanin, the tyr mutant was an albino lacking blue-black stripes with red eyes containing non-pigmented RPE; features which are similar to those of human OCA. The mutant was non-lethal and showed normal development until adulthood. The 5 dpf tyr mutant zebrafish larva did not demonstrate any color preference. In the color preference test, tyr mutants spent an equal amount of time in each color zone which was comparable to wild-type larvae in the sleeveless control maze. This finding suggests that tyrosinase $\mathrm{KO} \# 2 \mathrm{mu}$ tants have similar phenotypes to those of OCA patients; particularly in regards to the concurrence of color blindness in tyr mutants and OCA patients.

In this study, a simple color preference test in zebrafish was established, and using CRISPR/Cas9, a zebrafish OCA model which has hypopigmented RPE resulting in color blindness was generated. Color sensation of zebrafish is very unique, and especially $B$ over $Y$ color recognition is distinctive enough to be applied to various genetic and pharmaceutical studies (Supplementary Fig. 2). This color preference test will allow high- 
A

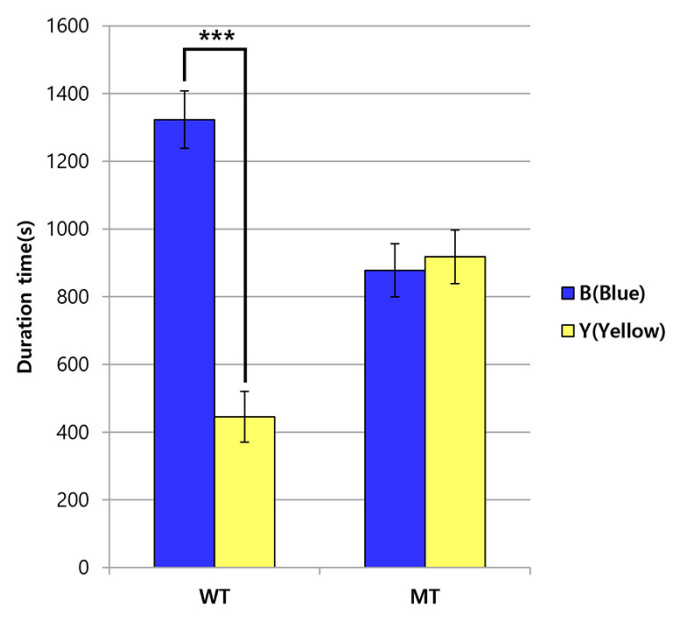

B

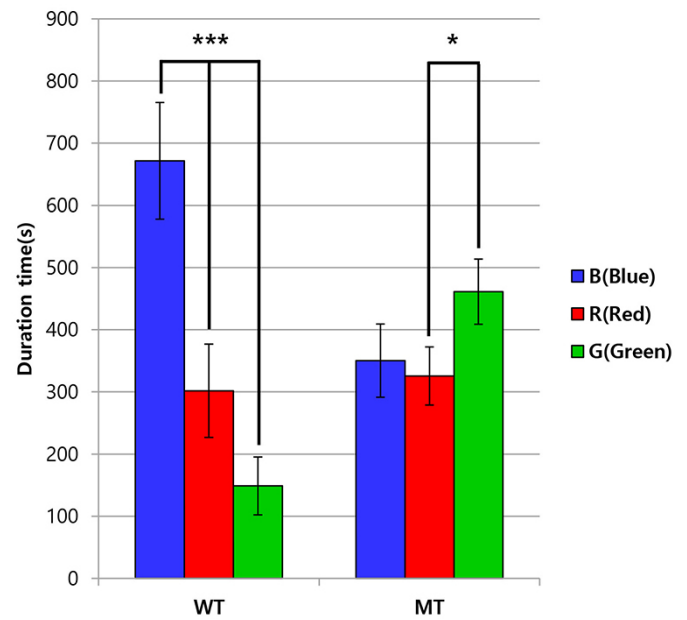

Fig. 6. Color preference test in a color cross maze with WT and MT. (A) Bidirectional color cross maze test using B and $Y$ color sleeves , WT $(5 \mathrm{dpf}, \mathrm{n}=20$ ) preferred $\mathrm{B}$ over $\mathrm{Y}$, staying in blue zone 3 times longer than in yellow zone. In contrast, MT ( $5 \mathrm{dpf}, \mathrm{n}=20)$ did not show significant preference between B and $Y$. (B) Cross color maze test using B, R and G color sleeves. WT ( $5 \mathrm{dpf}, \mathrm{n}=20)$ shows $B>R>G$ preferences, but MT ( $5 \mathrm{dpf}, \mathrm{n}=20)$ did not show any preferences between colors. In all panels, values represent means $\pm \mathrm{SD}\left({ }^{\star} \mathrm{p}<0.1 ;{ }^{* \star *} \mathrm{p}<0.01\right)$.

throughput screening studies to determine the quantitative effects of small molecules on color cognition and motor behavior.

Note: Supplementary information is available on the Molecules and Cells website (www.molcells.org).

\section{ACKNOWLEDGEMENTS}

This work was supported by National Research Foundation of Korea (NRF) grants funded by the Korean government Ministry of Science, ICT and Future Planning (MSIP) (2014R1A2A1A 11053562, 2015M3A9A8029261). The funders had no role in study design, data collection, and analysis, decision to publish, or preparation of the manuscript.

\section{REFERENCES}

Avdesh, A., Martin-Iverson, M.T., Mondal, A., Chen, M., Askraba, S., Morgan, N., Lardelli, M., Groth, D.M., Verdile, G., and Martins, R.N. (2012). Evaluation of color preference in zebrafish for learning and memory. J. Alzheimers. Dis. 28, 459-469.

Bowmaker, J.K. (2008). Evolution of vertebrate visual pigments. Vision Res. 48, 2022-2041.

Fadool, J.M., and Dowling, J.E. (2008). Zebrafish: a model system for the study of eye genetics. Prog. Retin. Eye Res. 27, 89-110.

Gerkema, M.P., Davies, W.I.L., Foster, R.G., Menaker, M., and Hut, R.a (2013). The nocturnal bottleneck and the evolution of activity patterns in mammals. Proc. Biol. Sci. 280, 20130508.

Glass, A.S., and Dahm, R. (2004). The zebrafish as a model organism for eye development. Ophthalmic Res. 36, 4-24.

Goldsmith, P., and Harris, W.A. (2003). The zebrafish as a tool for understanding the biology of visual disorders. Semin. Cell Dev. Biol. 14, 11-18.

Grønskov, K., Ek, J., and Brondum-Nielsen, K. (2007). Oculocutaneous albinism. Orphanet J. Rare Dis. 2, 43.

Jacobs, G.H. (2009). Evolution of colour vision in mammals. Philos. Trans. R. Soc. Lond. B. Biol. Sci. 364, 2957-2967.

Joselevitch, C., and Kamermans, M. (2009). Retinal parallel pathways: Seeing with our inner fish. Vision Res. 49, 943-959.

Lee, S.H., Lee, M.S., Choi, T.I., Hong, H., Seo, J.Y., Kim, C.H., and $\mathrm{Kim}, \mathrm{J}$. (2016). MCRS1 associates with cytoplasmic dynein and mediates pericentrosomal material recruitment. Sci. Rep. 6,
27284

Liu, N., Kong, X.D., Shi, H.R., Wu, Q.H., and Jiang, M. (2014). Tyrosinase gene mutations in the Chinese Han population with OCA1. Genet. Res. (Camb). 96, e14.

LoBue, V., and DeLoache, J.S. (2011). Pretty in pink: The early development of gender-stereotyped colour preferences. Br. J. Dev. Psychol. 29, 656-667.

May, M., Hwang, K.-S., Miles, J., Williams, C., Niranjan, T., Kahler, S.G., Chiurazzi, P., Steindl, K., Van, P.J., Spek, D., et al. (2015). $\mathrm{ZC} 4 \mathrm{H} 2$, an XLID gene, is required for the generation of a specific subset of CNS interneurons. Hum. Mol. Genet. 24, 4848-4861.

Miller, A.C., Voelker, L.H., Shah, A.N., and Moens, C.B. (2015) Neurobeachin is required postsynaptically for electrical and chemical synapse formation. Curr. Biol. 25, 16-28.

Nawrocki, L., BreMiller, R., Streisinger, G., and Kaplan, M. (1985). Larval and adult visual pigments of the zebrafish, Brachydanio rerio. Vision Res. 25, 1569-1576.

Orefice, L.L., Zimmerman, A.L., Chirila, A.M., Sleboda, S.J., Head, J.P., and Ginty, D.D. (2016). Peripheral mechanosensory neuron dysfunction underlies tactile and behavioral deficits in mouse models of ASDs article peripheral mechanosensory neuron dysfunction underlies tactile and behavioral deficits in mouse models of ASDs. Cell 166, 299-313.

Peeters, B.W.M.M., Moeskops, M., and Veenvliet, A.R.J. (2016). Color preference in Danio rerio: effects of age and anxiolytic treatments. Zebrafish 13, 330-334.

Richendrfer, H., Pelkowski, S.D., Colwill, R.M., and Creton, R. (2012). On the edge: pharmacological evidence for anxietyrelated behavior in zebrafish lavae. Behav. Brain Res. 228, 99106.

Robertson, D.S., McKenna, M.C., Toon, O.B., Hope, S., and Lillegraven, J.A. (2004). Survival in the first hours of the cenozoic. Bull. Geol. Soc. Am. 116, 760-768.

Singh, A.P., and Nüsslein-Volhard, C. (2015). Zebrafish stripes as a model for vertebrate colour pattern formation. Curr. Biol. 25, R81R92.

Sugimoto, M., Yuki, M., Miyakoshi, T., and Maruko, K. (2005). The influence of long-term chromatic adaptation on pigment cells and striped pigment patterns in the skin of the zebrafish,Danio rerio. $J$. Exp. Zool. Part A Comp. Exp. Biol. 303A, 430-440.

Sung, Y.H., Kim, J.M., Kim, H.T., Lee, J., Jeon, J., Jin, Y., Choi, J.H., Ban, Y.H., Ha, S.J., Kim, C.H., et al. (2014). Highly efficient gene knockout in mice and zebrafish with RNA-guided endonucleases. Genome Res. 24, 125-131. 